\title{
The Stability of Solutions for the Generalized Degasperis-Procesi Equation with Variable Coefficients
}

\author{
Jing Chen \\ School of Science, Southwest University of Science and Technology, Mianyang 621000, China \\ Correspondence should be addressed to Jing Chen; chenjing_math@163.com
}

Received 19 May 2015; Accepted 25 June 2015

Academic Editor: Xinguang Zhang

Copyright (C) 2015 Jing Chen. This is an open access article distributed under the Creative Commons Attribution License, which permits unrestricted use, distribution, and reproduction in any medium, provided the original work is properly cited.

A generalized Degasperis-Procesi equation with variable coefficients is investigated. The $L^{1}(R)$ stability of the strong solution for the equation is established under certain assumptions.

\section{Introduction}

The Degasperis-Procesi (DP) equation

$$
v_{t}-v_{t x x}+4 v v_{x}=3 v_{x} v_{x x}+v v_{x x x}, \quad t>0, x \in R,
$$

was discovered by Degasperis and Procesi [1] in a search for integrable equations similar to the Camassa-Holm equation. Degasperis and Procesi [1] studied a family of third order dispersive nonlinear equations

$$
v_{t}+v_{x}+\gamma v_{x x x}-\alpha^{2} v_{t x x}=\left(c_{1} v^{2}+c_{2} v_{x}^{2}+c_{3} v v_{x x}\right)_{x},
$$

where $\alpha, \gamma, c_{0}, c_{1}, c_{2}, c_{3} \in R$. It is found in [1] that there are only three equations that satisfy asymptotic integrability conditions within this family. By rescaling and applying a Galilean transformation, the three equations are Kortewegde Vries equation

$$
v_{t}+v_{x x x}+v v_{x}=0, \quad t>0, x \in R,
$$

the Camassa-Holm equation

$$
v_{t}-v_{t x x}+3 v v_{x}=2 v_{x} v_{x x}+v v_{x x x}, \quad t>0, x \in R,
$$

and the Degasperis-Procesi equation (1). Degasperis et al. [2] proved the formal integrability of (1) and the existence of the nonsmooth solutions by constructing a Lax pair.

In recent years, (1) which plays a similar role in water wave theory as the Camassa-Holm equation has caused extensive concern of many scholars (see [1-11]). For example,
Coclite and Karlsen [3] established the well-posedness of $L^{1} \cap B V$ weak solutions for (1). They proved uniqueness within a class of discontinuous solutions to (1) in [4]. Escher et al. [5] established the precise blow-up rate and proved the existence and uniqueness of global weak solutions to (1) in which the initial data satisfied appropriate conditions. Lai and $\mathrm{Wu}$ [7] investigated the local well-posedness of solutions to a generalization of both (1) and (4) in the Sobolev space $H^{s}(R)$ with $s>3 / 2$. Lenells [8] classified all weak traveling wave solutions of the Degasperis-Procesi equation (1). Ai and Gui [9] proved global existence of solutions for the viscous Degasperis-Procesi equation and showed that the blow-up phenomena occurs in finite time. Fu et al. [11] studied the orbital stability of the peakons for the Degasperis-Procesi equation with a strong dispersive term on the line and proved that the shapes of these peakons were stable under small perturbations.

As we know, their coefficients play an important role to study the fundamental dynamical properties of the Degasperis-Procesi models. It prompts us to study the following generalized Degasperis-Procesi equation:

$$
\begin{aligned}
v_{t}- & v_{t x x}+m(t, x) f^{\prime}(v) v_{x} \\
= & b(t, x)\left(f^{\prime}(v) v_{x x x}+3 f^{\prime \prime}(v) v_{x} v_{x x}+f^{\prime \prime \prime}(v) v_{x}^{3}\right) \\
& +2 b_{x}(t, x)\left(f^{\prime}(v) v_{x x}+f^{\prime \prime}(v) v_{x}^{2}\right) \\
& +b_{x x}(t, x) f^{\prime}(v) v_{x},
\end{aligned}
$$


where $m(t, x) \in C_{0}(R), b(t, x) \in C_{0}^{3}(R)$, and function $f(\cdot)$ is a polynomial of order $n(n \geq 2)$. Letting $m=4, b=1, f(v)=$ $v^{2} / 2$, (5) reduces to the Degasperis-Procesi equation (1). We consider the Cauchy problem of (5) with an initial condition $v_{0}(x)$. Namely,

$$
v(0, x)=v_{0}(x), \quad x \in R
$$

Assume that (5) possesses a bounded strong solution in its maximum existence time interval $[0, T)$ and $v_{0}$ lies in $L^{1}(R) \cap$ $H^{s}(R)(s>3 / 2)$. We use the approaches of Kružkov doubling the variables presented in [12] to prove the $L^{1}$ stability of the solution for the variable coefficients equation (5). From our knowledge, it has not been acquired in the literature.

This paper is organized as follows. Section 2 gives several lemmas. The proof of local solution stability is presented in Section 3.

\section{Preliminaries}

Applying the operator $\Lambda^{-2}=\left(1-\partial_{x}^{2}\right)^{-1}$ to (5), we obtain its equivalent form

$$
\begin{aligned}
v_{t} & +b(t, x) f^{\prime}(v) v_{x}+\Lambda^{-2}(m(t, x)-b(t, x)) \partial_{x} f(v) \\
& =0
\end{aligned}
$$

where $\Lambda^{-2} p(t, x)=\left(1-\partial_{x}^{2}\right)^{-1} p(t, x)=(1 / 2) \int_{R} e^{-|x-y|} p(t$, $y) d y$.

Equations (5) and (6) are equivalent to the problem

$$
\begin{aligned}
v_{t}+\partial_{x} P(t, x, v)+\Psi(t, x, v) & =0, \\
v(0, x) & =v_{0}(x),
\end{aligned}
$$

where $P(t, x, v)=b(t, x) f(v)$ and $\Psi(t, x, v)=\Lambda^{-2}(m(t, x)-$ $b(t, x)) \partial_{x} f(v)-b_{x}(t, x) f(v)$. Notice that $\partial_{x} P=P_{x}+P_{v} v_{x}$.

Remark. According to the statements presented in [7] or [12], we know that problem (8) has a unique local solution in the space $C\left([0, T), H^{s}(R)\right)$ if we assume $v_{0}(x) \in H^{s}(R)(s>3 / 2)$.

Assume that $v_{1}(t, x)$ and $v_{2}(t, x)$ are solutions of problem (8) in the domain $[0, T) \times R$ with initial functions $v_{10}(x)$ and $v_{20}(x) \in L^{1}(R) \cap H^{s}(R)(s>3 / 2)$, where $T$ is the maximum existence time of solutions. For simplicity, we denote by $c$ any positive constants. Now we give several lemmas.

Lemma 1. Let $v(t, x)$ be the solution of problem (8) and $\|v\|_{L^{\infty}(R)} \leq M$. Then

$$
\|\Psi(t, x, v)\|_{L^{\infty}(R)} \leq c M^{n},
$$

where positive constant $c$ depends on $\left\|v_{0}\right\|_{L^{\infty}}$ and $T$.
Proof. We have

$$
\begin{aligned}
|\Psi(t, x, v)| \\
=\left|\Lambda^{-2}(m(t, x)-b(t, x)) \partial_{x} f(v)-b_{x}(t, x) f(v)\right| \\
\leq\left|\frac{1}{2} \int_{R} e^{-|x-y|} m(t, y) \partial_{y} f(v) d y\right| \\
\quad+\left|\frac{1}{2} \int_{R} e^{-|x-y|} b(t, y) \partial_{y} f(v) d y\right|+\left|b_{x} f(v)\right| \\
\leq c\left|\int_{R} e^{-|x-y|} \operatorname{sign}(y-x) f(v) d y\right|+c|f(v)| \\
\leq c M^{n},
\end{aligned}
$$

in which we have used $\int_{R} e^{-|x-y|} d x=2$ to complete the proof.

Lemma 2. Assume that $v_{1}(t, x)$ and $v_{2}(t, x)$ are solutions of problem (8) in the domain $[0, T) \times R,\left\|v_{1}\right\|_{L^{\infty}(R)} \leq M$, and $\left\|v_{2}\right\|_{L^{\infty}(R)} \leq M$. Then

$$
\begin{aligned}
& \int_{-\infty}^{+\infty}\left|\Psi\left(t, x, v_{1}\right)-\Psi\left(t, x, v_{2}\right)\right| d x \\
& \quad \leq c \int_{-\infty}^{+\infty}\left|v_{1}-v_{2}\right| d x
\end{aligned}
$$

where $c>0$ depends on $\left\|v_{10}\right\|_{L^{\infty}(R)},\left\|v_{20}\right\|_{L^{\infty}(R)}$ and $T$.

Proof. Using the property of the operator $\Lambda^{-2}$, we get

$$
\begin{aligned}
& \int_{-\infty}^{+\infty}\left|\Psi\left(t, x, v_{1}\right)-\Psi\left(t, x, v_{2}\right)\right| d x \\
& \quad \leq \int_{-\infty}^{+\infty}\left|\Lambda^{-2}(m-b) \partial_{x}\left(f\left(v_{1}\right)-f\left(v_{2}\right)\right)\right| d x \\
& \quad+\int_{-\infty}^{+\infty}\left|\left(f\left(v_{1}\right)-f\left(v_{2}\right)\right)\right| d x \leq c \int_{-\infty}^{+\infty} d x \\
& \quad \cdot \int_{-\infty}^{+\infty}\left|e^{-|x-y|} \operatorname{sign}(y-x)\left(f\left(v_{1}\right)-f\left(v_{2}\right)\right)\right| d y \\
& +c \int_{-\infty}^{+\infty}\left|v_{1}-v_{2}\right| d x \leq c \int_{-\infty}^{+\infty}\left|f\left(v_{1}\right)-f\left(v_{2}\right)\right| d y \\
& \cdot \int_{-\infty}^{+\infty} e^{-|x-y|} d x+c \int_{-\infty}^{+\infty}\left|v_{1}-v_{2}\right| d x \\
& \quad \leq c \int_{-\infty}^{+\infty}\left|v_{1}-v_{2}\right| d x
\end{aligned}
$$

in which we apply the Tonelli Theorem to complete the proof.

Let $\delta(\sigma) \geq 0, \delta(\sigma) \equiv 0$, for $|\sigma| \geq 1 ; \int_{-\infty}^{+\infty} \delta(\sigma) d \sigma=$ 1 and $\delta(\sigma)$ is infinitely differential on $(-\infty,+\infty)$. Set 
$\delta_{\varepsilon}(\sigma)=\delta\left(\varepsilon^{-1} \sigma\right) / \varepsilon$, where $\varepsilon$ is an arbitrary positive constant. It is found that $\delta_{\varepsilon}(\sigma) \in C_{0}^{\infty}(-\infty,+\infty)$ and

$$
\begin{aligned}
\delta_{\varepsilon}(\sigma) & \geq 0, \\
\delta_{\varepsilon}(\sigma) & =0 \\
\left|\delta_{\varepsilon}(\sigma)\right| \leq \frac{c}{\varepsilon} & \\
\int_{-\infty}^{+\infty} \delta_{\varepsilon}(\sigma) d \sigma & =1
\end{aligned}
$$$$
\text { for }|\sigma| \geq \varepsilon \text {, }
$$

Let the function $\phi(x)$ be defined and locally integrable on $(-\infty,+\infty)$. Set $\phi^{\varepsilon}(x)$; denote the approximation function of $\phi(x)$ as

$$
\phi^{\varepsilon}(x)=\frac{1}{\varepsilon} \int_{-\infty}^{+\infty} \delta\left(\frac{x-y}{\varepsilon}\right) \phi(y) d y .
$$

We call $x_{0}$ a Lebesgue point of the function $\phi(x)$ if

$$
\lim _{\varepsilon \rightarrow 0} \frac{1}{\varepsilon} \int_{x_{0}-\varepsilon}^{x_{0}+\varepsilon}\left|\phi(x)-\phi\left(x_{0}\right)\right| d x=0 .
$$

At any Lebesgue point $x_{0}$, we get

$$
\lim _{\varepsilon \rightarrow 0} \phi^{\varepsilon}\left(x_{0}\right)=\phi\left(x_{0}\right) \text {. }
$$

Since the set of points which are not Lebesgue points of $\phi(x)$ has measure zero, we have $\phi^{\varepsilon}(x) \rightarrow \phi(x)$ as $\varepsilon \rightarrow 0$ almost everywhere.

For any $T>0$, we denote the band $\{(t, x):[0, T] \times R\}$ by $\omega_{T}$. Let $K_{r}=\{x:|x| \leq r\}$ and

$$
\begin{aligned}
\Pi & =\left\{(t, x, \tau, y):\left|\frac{t-\tau}{2}\right| \leq \varepsilon, \rho \leq \frac{t+\tau}{2} \leq T\right. \\
& \left.-\rho,\left|\frac{x-y}{2}\right| \leq \varepsilon,\left|\frac{x+y}{2}\right| \leq r-\rho\right\},
\end{aligned}
$$

where $r>0, \rho>0$.

We state the concept of a characteristic cone. Let $\|v\|_{L^{\infty}(R)} \leq M$, for any $R_{1}>0$; we define

$$
N>\max _{(t, x) \in[0, T] \times K_{R_{1}}}\left|f^{\prime}(v)\right| .
$$

Let $\Omega$ represent the cone $\left\{(t, x):|x| \leq R_{1}-N t, 0 \leq t \leq T_{1}=\right.$ $\left.\min \left(T, R_{1} N^{-1}\right)\right\}$ and let $S_{\tau}$ designate the cross section of the cone $\Omega$ by the plane $t=\tau, \tau \in\left[0, T_{1}\right]$.

Lemma 3 (see [12]). Let the function $\phi(t, x)$ be bounded and measurable in cylinder $\left[0, T_{1}\right] \times K_{r}$. For any $\rho \in\left(0, \min \left[r, T_{1}\right]\right)$ and any $\varepsilon \in(0, \rho)$, the function

$$
V_{\varepsilon}=\frac{1}{\varepsilon^{2}} \iiint \int_{\Pi}|\phi(t, x)-\phi(\tau, y)| d t d x d \tau d y
$$

satisfies

$$
\lim _{\varepsilon \rightarrow 0} V_{\varepsilon}=0
$$

Lemma 4 (see [12]). If $\partial F(\phi) / \partial \phi$ is bounded, the function $H(\phi, \psi)=\operatorname{sign}(\phi-\psi)(F(\phi)-F(\psi))$ satisfies the Lipschitz condition in $\phi$ and $\psi$.

Lemma 5. If $v(t, x)$ is the solution of problem (11) on $\omega_{T}$, $\eta(t, x) \in C_{0}^{\infty}\left(\omega_{T}\right)$, it holds that

$$
\begin{aligned}
& \iint_{\omega_{T}}\left\{|v-k| \eta_{t}\right. \\
& \quad+\operatorname{sign}(v-k)[P(t, x, v)-P(t, x, k)] \eta_{x} \\
& \left.\quad-\operatorname{sign}(v-k)\left(P_{x}(t, x, k)+\Psi(t, x, v)\right) \eta\right\} d t d x \\
& \quad=0,
\end{aligned}
$$

where $k$ is an arbitrary constant.

Proof. Suppose that $\Phi(v)$ is a twice differential function. Multiplying the first equation of problem (8) by $\Phi^{\prime}(v) \eta(t, x)$ and integrating over $\omega_{T}$, we get

$$
\begin{gathered}
\iint_{\omega_{T}}\left\{\Phi^{\prime}(v) \eta v_{t}+\Phi^{\prime}(v) \eta P_{x}+\Phi^{\prime}(v) \eta P_{v} v_{x}\right. \\
\left.+\Phi^{\prime}(v) \eta \Psi(t, x, v)\right\} d t d x=0 .
\end{gathered}
$$

Using the method of integration by parts, we get

$$
\iint_{\omega_{T}} \Phi^{\prime}(v) \eta v_{t} d t d x=-\iint_{\varrho_{T}} \Phi(v) \eta_{t} d t d x
$$

Notice that

$$
\begin{aligned}
& \left(\int_{k}^{v} \Phi^{\prime}(z) P_{z}(t, x, z) d z\right)_{x}^{\prime} \\
& \quad=\Phi^{\prime}(v) P_{v}(v) v_{x} \\
& \quad+\int_{k}^{v}\left(\Phi^{\prime \prime}(z) P_{z} z_{x}+\Phi^{\prime}(z) P_{z x}\right) d z .
\end{aligned}
$$

So

$$
\begin{aligned}
\int_{-\infty}^{+\infty} & \left(\int_{k}^{v} \Phi^{\prime}(z) P_{z} d z\right) \eta_{x} d x \\
= & -\int_{-\infty}^{+\infty} \Phi^{\prime}(v) P_{v} v_{x} \eta d x \\
& -\int_{-\infty}^{+\infty}\left[\int_{k}^{v}\left(\Phi^{\prime \prime}(z) P_{z} z_{x}+\Phi^{\prime}(z) P_{z x}\right) d z\right] \eta d x .
\end{aligned}
$$

Then we have

$$
\begin{aligned}
& \iint_{\omega_{T}} \Phi^{\prime}(v) P_{v} v_{x} \eta d t d x=-\iint_{\omega_{T}}\left(\int_{k}^{v} v^{\prime}(z) P_{z} d z\right) \\
& \quad \cdot \eta_{x} d t d x \\
& \quad+\iint_{\omega_{T}}\left[\int_{k}^{v}\left(\Phi^{\prime \prime}(z) P_{z} z_{x}+\Phi^{\prime}(z) P_{z x}\right) d z\right] \eta d t d x .
\end{aligned}
$$


Substituting (23) and (26) into (22), we get

$$
\begin{aligned}
& \iint_{\omega_{T}}\left\{\Phi^{\prime}(v) \eta_{t}+\left(\int_{k}^{v} \Phi^{\prime}(z) P_{z} d z\right) \eta_{x}\right. \\
& \quad+\left(\int_{k}^{v} \Phi^{\prime \prime}(z) P_{z} z_{x} d z\right) \eta+\left(\int_{k}^{v} \Phi^{\prime}(z) P_{z x} d z\right) \eta \\
& \left.-\Phi^{\prime}(v) \eta P_{x}-\Phi^{\prime}(v) \eta \Psi\right\} d t d x=0 .
\end{aligned}
$$

Let $\Phi^{\varepsilon}(v)$ be an approximation of the function $|v-k|$. When $\varepsilon \rightarrow 0, \Phi^{\varepsilon}(v) \rightarrow \Phi(v)$. Setting $\Phi(v)=|v-k|$, then $\Phi^{\prime}(v)=$ $\operatorname{sign}(v-k), \Phi^{\prime \prime}(v)=0$. Hence,

$$
\begin{aligned}
& \int_{k}^{v} \Phi^{\prime}(z) P_{z} d z \\
& \quad=\operatorname{sign}(v-k)(P(t, x, v)-P(t, x, k)), \\
& \int_{k}^{v} \Phi^{\prime}(z) P_{z x} d z \\
& \quad=\operatorname{sign}(v-k)\left(P_{x}(t, x, v)-P_{x}(t, x, k)\right),
\end{aligned}
$$

combining with (27), we complete the proof.

\section{Main Result}

Set function $\eta(t, x) \in C_{0}^{\infty}\left(\omega_{T}\right), \eta(t, x) \equiv 0$, outside the cylinder $\{(t, x)\}=[\rho, T-2 \rho] \times K_{r-2 \rho}$, where $K_{r-2 \rho}=\{|x|$ : $|x| \leq r-2 \rho\}, r>0,0<2 \rho<\min (T, r)$. Now we give the main result of this work.

Theorem 6. Assume that $v_{1}(t, x)$ and $v_{2}(t, x)$ are two strong solutions of problem (8) with initial data $v_{10}(x), v_{20}(x) \epsilon$ $L^{1}(R) \cap H^{s}(R)(s>3 / 2)$. Let $T>0$ be the maximum existence time of $v_{1}(t, x)$ and $v_{2}(t, x)$. If $\left\|v_{1}(t, x)\right\|_{L^{\infty}} \leq M$ and $\left\|v_{2}(t, x)\right\|_{L^{\infty}} \leq M$, for any $t \in[0, T]$, it holds that

$$
\begin{aligned}
& \left\|v_{1}(t, x)-v_{2}(t, x)\right\|_{L^{1}} \\
& \quad \leq e^{c t} \int_{-\infty}^{+\infty}\left|v_{10}(x)-v_{20}(x)\right| d x,
\end{aligned}
$$

where $c$ is a positive constant depending on $\left\|v_{10}\right\|_{L^{\infty}(R)}$ and $\left\|v_{20}\right\|_{L^{\infty}(R)}$.

Proof. Set $k=\psi(\tau, y), \eta(t, x)=h(t, x, \tau, y)$. Using the Kružkov device of doubling the variables in [12] and Lemma 5, we get

$$
\begin{aligned}
& \iiint \int_{\omega_{T} \times \emptyset_{T}}\left\{\left|v_{1}(t, x)-v_{2}(\tau, y)\right| h_{t}\right. \\
& \quad+\operatorname{sign}\left(v_{1}(t, x)-v_{2}(\tau, y)\right) \\
& \quad \cdot\left[P\left(t, x, v_{1}(t, x)\right)-P\left(\tau, y, v_{2}(\tau, y)\right)\right] h_{x} \\
& \quad-\operatorname{sign}\left(v_{1}(t, x)-v_{2}(\tau, y)\right) \\
& \left.\quad \cdot\left[P_{x}\left(t, x, v_{2}(\tau, y)\right)+\Psi\left(t, x, v_{1}(t, x)\right)\right] h\right\} d t d x d \tau d y \\
& \quad=0 .
\end{aligned}
$$

Similarly, we have

$$
\begin{aligned}
& \iiint \int_{\omega_{T} \times \oplus_{T}}\left\{\left|v_{2}(\tau, y)-v_{1}(t, x)\right| h_{\tau}\right. \\
& \quad+\operatorname{sign}\left(v_{2}(\tau, y)-v_{1}(t, x)\right) \\
& \quad \cdot\left[P\left(\tau, y, v_{2}(\tau, y)\right)-P\left(t, x, v_{1}(t, x)\right)\right] h_{y} \\
& \quad-\operatorname{sign}\left(v_{2}(\tau, y)-v_{1}(t, x)\right) \\
& \left.\quad \cdot\left[P_{y}\left(\tau, y, v_{1}(t, x)\right)+\Psi\left(\tau, y, v_{2}(\tau, y)\right)\right] h\right\} d t d x d \tau d y \\
& \quad=0 .
\end{aligned}
$$

Adding (31) and (32), we obtain

$$
\begin{aligned}
& \iiint \int_{\omega_{T} \times \omega_{T}}\left\{\left|v_{1}(t, x)-v_{2}(\tau, y)\right|\left(h_{t}+h_{\tau}\right)\right. \\
& \quad+\operatorname{sign}\left(v_{1}(t, x)-v_{2}(\tau, y)\right)\left[P\left(t, x, v_{1}(t, x)\right)\right. \\
& \left.\quad-P\left(t, x, v_{2}(\tau, y)\right)\right]\left(h_{x}+h_{y}\right) \\
& +\operatorname{sign}\left(v_{1}(t, x)-v_{2}(\tau, y)\right) \\
& +\left(\left[P\left(\tau, y, v_{2}(\tau, y)\right)-P\left(t, x, v_{2}(\tau, y)\right)\right] h_{x}\right. \\
& +\left[P\left(\tau, y, v_{1}(t, x)\right)-P\left(t, x, v_{1}(t, x)\right)\right] h_{y} \\
& \left.+\left[P P_{y}\left(\tau, y, v_{1}(t, x)\right)-P_{x}\left(t, x, v_{2}(\tau, y)\right)\right] h\right) \\
& \quad-\operatorname{sign}\left(v_{1}(t, x)-v_{2}(\tau, y)\right)\left[\Psi\left(t, x, v_{1}(t, x)\right)\right. \\
& \left.\left.\quad-\Psi\left(\tau, y, v_{2}(\tau, y)\right)\right] h\right\} d t d x d \tau d y \\
& \quad=\iiint \int_{\omega_{T} \times \omega_{T}}\left(A,+A_{2}+A_{3}+A_{4}\right) d t d x d \tau d y \\
& =0 .
\end{aligned}
$$

Set function

$$
\begin{aligned}
& h(t, x, \tau, y) \\
& \quad=\eta\left(\frac{t+\tau}{2}, \frac{x+y}{2}\right) \delta_{\varepsilon}\left(\frac{t-\tau}{2}\right) \delta_{\varepsilon}\left(\frac{x-y}{2}\right) \\
& =\eta(\cdots) \lambda_{\varepsilon}(\vdots)
\end{aligned}
$$

in which $(\cdots)=((t+\tau) / 2,(x+y) / 2)$ and $(\vdots)=((t-\tau) / 2,(x-$ $y) / 2$ ). Thus, we obtain

$$
\begin{aligned}
& h_{t}+h_{\tau}=\eta_{t}(\cdots) \lambda_{\varepsilon}(\vdots), \\
& h_{x}+h_{y}=\eta_{x}(\cdots) \lambda_{\varepsilon}(\vdots) .
\end{aligned}
$$

We will prove that the form $A_{3}$ in (33) approaches zero as $\varepsilon \rightarrow 0$. In fact, the coefficients of $\eta_{x}$ and $\eta_{y}$ in $A_{3}$ vanish for 
$|t-\tau|+|x-y|=0$. Thus the integrals of $A_{3}$ can be rewritten as the following concrete form:

$$
\begin{aligned}
& \iiint \int_{\omega_{T} \times \omega_{T}} \operatorname{sign}\left(v_{1}(t, x)-v_{2}(\tau, y)\right) \\
& \quad \cdot\left\{\left[P\left(\tau, y, v_{2}(\tau, y)\right)-P\left(t, x, v_{2}(\tau, y)\right)\right]\left(\lambda_{\varepsilon}\right)_{x}\right. \\
& \quad-P_{x}\left(t, x, v_{2}(\tau, y)\right) \lambda_{\varepsilon} \\
& \quad+\left[P\left(\tau, y, v_{1}(t, x)\right)-P\left(t, x, v_{1}(t, x)\right)\right]\left(\lambda_{\varepsilon}\right)_{y} \\
& \left.\quad+P_{y}\left(\tau, y, v_{1}(t, x)\right) \lambda_{\varepsilon}\right\} \eta(\cdots) d t d x d \tau d y=A_{\varepsilon} .
\end{aligned}
$$

In the following computations, we omit the index $\varepsilon$ of function $\lambda$. Applying the Taylor formula, we have the relations

$$
\begin{aligned}
& {\left[P\left(\tau, y, v_{2}(\tau, y)\right)-P\left(t, x, v_{2}(\tau, y)\right)\right] \lambda_{x}} \\
& \quad-P_{x}\left(t, x, v_{2}(\tau, y)\right) \lambda \\
& \quad=\left(\left[P\left(\tau, y, v_{2}(\tau, y)\right)-P\left(t, x, v_{2}(\tau, y)\right)\right] \lambda\right)_{x} \\
& \quad=P_{\tau}\left(\tau, y, v_{2}(\tau, y)\right)(\tau-t) \lambda_{x} \\
& \quad+P_{y}\left(\tau, y, v_{2}(\tau, y)\right)(y-x) \lambda_{x} \\
& \quad-P_{y}\left(\tau, y, v_{2}(\tau, y)\right) \lambda+\alpha_{1} \lambda_{x}+\alpha_{0} \lambda \\
& \quad=P_{\tau}\left(\tau, y, v_{2}(\tau, y)\right)((\tau-t) \lambda)_{x} \\
& \quad+P_{y}\left(\tau, y, v_{2}(\tau, y)\right)((y-x) \lambda)_{x}+\alpha_{1} \lambda_{x}+\alpha_{0} \lambda .
\end{aligned}
$$

It is seen that the identity $\lambda_{y}=-\lambda_{x}$. In a similar way, we obtain

$$
\begin{aligned}
& {\left[P\left(\tau, y, v_{1}(t, x)\right)-P\left(t, x, v_{1}(t, x)\right)\right] \lambda_{y}} \\
& \quad+P_{y}\left(\tau, y, v_{1}(t, x)\right) \lambda \\
& \quad=P_{\tau}\left(\tau, y, v_{1}(t, x)\right)(\tau-t) \lambda_{y} \\
& \quad+P_{y}\left(\tau, y, v_{1}(t, x)\right)(y-x) \lambda_{y} \\
& \quad+P_{y}\left(\tau, y, v_{1}(t, x)\right) \lambda+\beta_{1} \lambda_{y} \\
& \quad=P_{\tau}\left(\tau, y, v_{1}(t, x)\right)((t-\tau) \lambda)_{x} \\
& \quad+P_{y}\left(\tau, y, v_{1}(t, x)\right)((x-y) \lambda)_{x}+\beta_{1} \lambda_{y} .
\end{aligned}
$$

The functions $\alpha_{0}, \alpha_{1}$, and $\beta_{1}$ in (37) and (38) satisfy

$$
\left|\alpha_{0}\right|+\left|\alpha_{1}\right|+\left|\beta_{1}\right| \leq d \varepsilon(d),
$$

where $d=|t-\tau|+|x-y|$ and $\varepsilon(d) \rightarrow 0$ as $d \rightarrow 0$. There are $\lambda=\lambda_{\varepsilon} \equiv 0$ for $|t-\tau| \geq 2 \varepsilon$ or $|x-y| \geq 2 \varepsilon$ and

$$
\begin{gathered}
\left|\lambda_{x}\right|+\left|\lambda_{y}\right| \leq \frac{c}{\varepsilon^{3}}, \\
|\eta(\cdots)-\eta(\tau, y)| \leq c(|t-\tau|+|x-y|) .
\end{gathered}
$$

Hence, we get

$$
\begin{aligned}
A_{\varepsilon} & =\iiint \int_{\omega_{T} \times \omega_{T}} \operatorname{sign}\left(v_{1}(t, x)-v_{2}(\tau, y)\right) \\
& \cdot\left\{\left[P_{\tau}\left(\tau, y, v_{2}(\tau, y)\right)-P_{\tau}\left(t, x, v_{2}(\tau, y)\right)\right]\right. \\
& \cdot((\tau-t) \lambda)_{x} \\
& +\left[P_{y}\left(\tau, y, v_{2}(t, x)\right)-P_{y}\left(\tau, y, v_{1}(t, x)\right)\right] \\
& \left.\cdot((y-x) \lambda)_{x}\right\} \eta(\tau, y) d t d x d \tau d y+I(\varepsilon),
\end{aligned}
$$

where $I(\varepsilon) \rightarrow 0$ as $\varepsilon \rightarrow 0$.

We denote the integrand in (41) as

$$
\begin{aligned}
B_{\varepsilon} & =K_{1}\left(\tau, y, v_{1}(t, x), v_{2}(\tau, y)\right)((\tau-t) \eta(\tau, y) \lambda)_{x} \\
& +K_{2}\left(\tau, y, v_{1}(t, x), v_{2}(\tau, y)\right)((y-x) \eta(\tau, y) \lambda)_{x} \\
& =\left\{K_{1}\left(\tau, y, v_{1}(t, x), v_{2}(\tau, y)\right)\right. \\
& \left.-K_{1}\left(\tau, y, v_{1}(\tau, y), v_{2}(\tau, y)\right)\right\}((\tau-t) \eta(\tau, y) \lambda)_{x} \\
& +\left\{K_{2}\left(\tau, y, v_{1}(t, x), v_{2}(\tau, y)\right)\right. \\
& \left.-K_{2}\left(\tau, y, v_{1}(\tau, y), v_{2}(\tau, y)\right)\right\} \\
& \cdot((y-x) \eta(\tau, y) \lambda)_{x} \\
& +K_{1}\left(\tau, y, v_{1}(\tau, y), v_{2}(\tau, y)\right)((\tau-t) \eta(\tau, y) \lambda)_{x} \\
& +K_{2}\left(\tau, y, v_{1}(\tau, y), v_{2}(\tau, y)\right)((y-x) \eta(\tau, y) \lambda)_{x},
\end{aligned}
$$

where $K_{1}$ and $K_{2}$ satisfy the Lipschitz condition in $v$. Applying the property of the function $\eta(\tau, y) \lambda_{\varepsilon}$ and the method of integration by parts, we have

$$
\begin{aligned}
& \iiint \int_{\omega_{T} \times \omega_{T}}\left\{K_{1}\left(\tau, y, v_{1}(\tau, y), v_{2}(\tau, y)\right)\right. \\
& \quad \cdot((\tau-t) \eta(\tau, y) \lambda)_{x} \\
& \quad+K_{2}\left(\tau, y, v_{1}(\tau, y), v_{2}(\tau, y)\right) \\
& \left.\quad \cdot((y-x) \eta(\tau, y) \lambda)_{x}\right\} d t d x d \tau d y=0 .
\end{aligned}
$$

Hence

$$
\begin{aligned}
& \left|A_{\varepsilon}-I(\varepsilon)\right|=\left|\iiint \int_{\omega_{T} \times \omega_{T}} B_{\varepsilon} d t d x d \tau d y\right| \leq c \\
& \cdot \iiint \int_{\omega_{T} \times \omega_{T}}\left|v_{1}(t, x)-v_{2}(\tau, y)\right| \\
& \cdot\left\{(|\tau-t|+|y-x|) \eta(\tau, y)\left|\lambda_{x}\right|+\lambda_{\varepsilon}\right\} d t d x d \tau d y \\
& \quad \leq \frac{c}{\varepsilon^{2}} \iiint \int_{\Pi}\left|v_{1}(t, x)-v_{2}(\tau, y)\right| d t d x d \tau d y .
\end{aligned}
$$

Using Lemma 3, $A_{\varepsilon}-I(\varepsilon) \rightarrow 0$ as $\varepsilon \rightarrow 0$. Therefore, we have

$$
\lim _{\varepsilon \rightarrow 0} \iiint \int_{\omega_{T} \times \omega_{T}} A_{3} d t d x d \tau d y=0 .
$$


It follows from (33) to (45) that

$$
\begin{aligned}
& \iiint \int_{\omega_{T} \times \omega_{T}}\left\{\left|v_{1}(t, x)-v_{2}(\tau, y)\right| \eta_{t}\right. \\
& \quad+\operatorname{sign}\left(v_{1}(t, x)-v_{2}(\tau, y)\right) \\
& \left.\quad \cdot\left[P\left(t, x, v_{1}(t, x)\right)-P\left(t, x, v_{2}(\tau, y)\right)\right] \eta_{x}\right\} \\
& \quad \cdot \lambda_{\varepsilon} d t d x d \tau d y \\
& \quad+\mid \iiint \int_{\omega_{T} \times \omega_{T}} \operatorname{sign}\left(v_{1}(t, x)-v_{2}(\tau, y)\right) \\
& \quad \cdot\left[\Psi\left(t, x, v_{1}(t, x)\right)-\Psi\left(\tau, y, v_{2}(\tau, y)\right)\right] \\
& \quad \cdot \eta \lambda_{\varepsilon} d t d x d \tau d y \mid \geq 0 .
\end{aligned}
$$

We note that the first two terms of the integrand of (46) have the form

$$
F_{\varepsilon}=F\left(t, x, \tau, y, v_{1}(t, x), v_{2}(\tau, y)\right) \lambda_{\varepsilon}(\vdots),
$$

where $F$ satisfies the Lipschitz condition in all its variables. Then

$$
\begin{aligned}
& \iiint \int_{\omega_{T} \times \omega_{T}} F_{\varepsilon} d t d x d \tau d y \\
& \quad=\iiint \int_{\omega_{T} \times \omega_{T}} F\left(t, x, \tau, y, v_{1}(t, x), v_{2}(\tau, y)\right) \\
& \quad \cdot \lambda_{\varepsilon} d t d x d \tau d y \\
& \quad=\iiint \int_{\omega_{T} \times \omega_{T}}\left\{F\left(t, x, \tau, y, v_{1}(t, x), v_{2}(\tau, y)\right)\right. \\
& \left.\quad-F\left(t, x, t, x, v_{1}(t, x), v_{2}(t, x)\right)\right\} \lambda_{\varepsilon} d t d x d \tau d y \\
& \quad+\iiint \int_{\omega_{T} \times \omega_{T}} F\left(t, x, t, x, v_{1}(t, x), v_{2}(t, x)\right) \\
& \quad \cdot \lambda_{\varepsilon} d t d x d \tau d y=J_{11}(\varepsilon)+J_{12} .
\end{aligned}
$$

Note that $F_{\varepsilon}=0$ outside the region $\Pi$. Applying the estimate $\left|\lambda_{\varepsilon}(:)\right| \leq c / \varepsilon^{2}$ and Lemma 4 , we get

$$
\begin{aligned}
& \left|J_{11}(\varepsilon)\right| \\
& \quad \leq c\left[\varepsilon+\frac{1}{\varepsilon^{2}} \iiint \int_{\Pi}\left|v_{1}(t, x)-v_{2}(\tau, y)\right| d t d x d \tau d y\right],
\end{aligned}
$$

where $c$ is a positive constant independent of $\varepsilon$. Using Lemma 3, we know $J_{11}(\varepsilon) \rightarrow 0$ as $\varepsilon \rightarrow 0$.

For the term $J_{12}$, we substitute $t=\alpha,(t-\tau) / 2=\beta, x=\zeta$, $(x-y) / 2=\gamma$. Combining with the identity

$$
\int_{-\varepsilon}^{\varepsilon} \int_{-\infty}^{+\infty} \lambda_{\varepsilon}(\beta, \gamma) d \beta d \gamma=1
$$

we obtain

$$
\begin{aligned}
J_{12} & =2^{2} \iint_{\omega_{T} \times \omega_{T}} F(\alpha, \zeta, \alpha, \zeta, \phi(\alpha, \zeta), \psi(\alpha, \zeta)) \\
& \cdot\left(\int_{-\varepsilon}^{\varepsilon} \int_{-\infty}^{+\infty} \lambda_{\varepsilon}(\beta, \gamma) d \beta d \gamma\right) d \alpha d \zeta=4 \\
& \cdot \iint_{\omega_{T}} F\left(t, x, t, x, v_{1}(t, x), v_{2}(t, x)\right) d t d x .
\end{aligned}
$$

Thus, we have

$$
\begin{aligned}
& \lim _{\varepsilon \rightarrow 0} \iiint \int_{\omega_{T} \times \omega_{T}} F_{\varepsilon} \\
& \quad=4 \iint_{\omega_{T}} F\left(t, x, t, x, v_{1}(t, x), v_{2}(t, x)\right) d t d x .
\end{aligned}
$$

Similarly, the integrand of the third term in (46) can be represented as

$$
\begin{aligned}
\bar{F}_{\varepsilon} & =\operatorname{sign}\left(v_{1}(t, x)-v_{2}(\tau, y)\right) \\
\cdot & {\left[\Psi\left(t, x, v_{1}(t, x)\right)-\Psi\left(\tau, y, v_{2}(\tau, y)\right)\right] g \lambda_{\varepsilon} } \\
& =\bar{F}\left(t, x, \tau, y, v_{1}(t, x), v_{2}(\tau, y)\right) \lambda_{\varepsilon}(\vdots)
\end{aligned}
$$

Then

$$
\begin{aligned}
& \iiint \int_{\omega_{T} \times \omega_{T}} \bar{F}_{\varepsilon} d t d x d \tau d y \\
& \quad=\iiint \int_{\omega_{T} \times \omega_{T}}\left\{\bar{F}\left(t, x, \tau, y, v_{1}(t, x), v_{2}(\tau, y)\right)\right. \\
& \left.\quad-\bar{F}\left(t, x, t, x, v_{1}(t, x), v_{2}(t, x)\right) \lambda_{\varepsilon}\right\} d t d x d \tau d y \\
& \quad+\iiint \int_{\omega_{T} \times \omega_{T}} \bar{F}\left(t, x, t, x, v_{1}(t, x), v_{2}(t, x)\right) \\
& \quad \cdot \lambda_{\varepsilon} d t d x d \tau d y=J_{21}(\varepsilon)+J_{22} .
\end{aligned}
$$

It holds that

$$
\begin{aligned}
& \left|J_{21}(\varepsilon)\right| \\
& \quad \leq c\left[\varepsilon+\frac{1}{\varepsilon^{2}} \iiint \int_{\Pi}\left|v_{1}(t, x)-v_{2}(\tau, y)\right| d t d x d \tau d y\right] .
\end{aligned}
$$

Using Lemma 3, it yields $J_{21}(\varepsilon) \rightarrow 0$ as $\varepsilon \rightarrow 0$. Repeating the steps as before, we have

$$
J_{22}=4 \iint_{\omega_{T}} \bar{F}\left(t, x, t, x, v_{1}(t, x), v_{2}(t, x)\right) d t d x
$$


From (46) to (56), we get

$$
\begin{aligned}
& \iint_{\omega_{T}}\left\{\left|v_{1}(t, x)-v_{2}(t, x)\right| \eta_{t}\right. \\
& \quad+\operatorname{sign}\left(v_{1}(t, x)-v_{2}(t, x)\right) \\
& \left.\quad \cdot\left[P\left(t, x, v_{1}(t, x)\right)-P\left(\tau, y, v_{2}(\tau, y)\right)\right] \eta_{x}\right\} d t d x \\
& \quad+\mid \iint_{\omega_{T}} \operatorname{sign}\left(v_{1}(t, x)-v_{2}(t, x)\right) \\
& \quad \cdot\left[\Psi\left(t, x, v_{1}\right)-\Psi\left(t, x, v_{2}\right)\right] \eta d t d x \mid \geq 0 .
\end{aligned}
$$

We set

$$
\begin{aligned}
g(t) & =\int_{-\infty}^{+\infty}\left|v_{1}(t, x)-v_{2}(t, x)\right| d x, \\
\mu_{\varepsilon}(\sigma) & =\int_{-\infty}^{\sigma} \delta_{\varepsilon}(\sigma) d \sigma .
\end{aligned}
$$

Take two numbers $\rho, \tau \in\left(0, T_{1}\right)$ and $\rho<\tau$. In (57), we set

$$
\begin{aligned}
\eta(t, x)=\left[\mu_{\varepsilon}(t-\rho)-\mu_{\varepsilon}(t-\tau)\right] & \chi(t, x), \\
& \varepsilon<\min \left(\rho, T_{1}-\tau\right),
\end{aligned}
$$

in which

$$
\chi(t, x)=\chi_{\theta}(t, x)=1-\mu_{\theta}\left(|x|+N t-R_{1}+\theta\right),
$$

where $\theta$ is a small positive constant and $\chi(t, x)=0$ outside the cone $\Omega$. When $\theta \rightarrow 0, R_{1} \rightarrow+\infty$, we observe that $\chi_{\theta} \rightarrow 1$. By the definition of the number $N$, we have

$$
0=\chi_{t}+N\left|\chi_{x}\right| \geq \chi_{t}+N \chi_{x}, \quad(t, x) \in \Omega .
$$

Applying (57)-(60), we get

$$
\begin{aligned}
& \iint_{\omega_{T_{1}}}\left\{\left|v_{1}(t, x)-v_{2}(t, x)\right|\left[\delta_{\varepsilon}(t-\rho)-\delta_{\varepsilon}(t-\tau)\right]\right. \\
& \left.\cdot \chi_{\theta}(t, x)\right\} d t d x+\int_{0}^{T_{1}} d t \\
& \quad \cdot \int_{-\infty}^{+\infty}\left\{\left|\Psi\left(t, x, v_{1}\right)-\Psi\left(t, x, v_{2}\right)\right|\right. \\
& \left.\cdot\left[\mu_{\varepsilon}(t-\rho)-\mu_{\varepsilon}(t-\tau)\right] \chi_{\theta}(t, x)\right\} d x \geq 0 .
\end{aligned}
$$

In (62), sending $\theta \rightarrow 0, R_{1} \rightarrow+\infty$ and using Lemma 2 , we obtain

$$
\begin{aligned}
& \int_{0}^{T_{1}}\left[\delta_{\varepsilon}(t-\rho)-\delta_{\varepsilon}(t-\tau)\right] h(t) d t \\
& \quad+c \int_{0}^{T_{1}}\left[\mu_{\varepsilon}(t-\rho)-\mu_{\varepsilon}(t-\tau)\right] g(t) d t \geq 0,
\end{aligned}
$$

where $c$ is independent of $\varepsilon$.
Applying the properties of the function $\delta_{\varepsilon}$ for $\varepsilon \leq \min (\rho$, $\left.T_{1}-\rho\right)$, we get

$$
\begin{aligned}
& \left|\int_{0}^{T_{1}} \delta_{\varepsilon}(t-\rho) g(t)-g(\rho) d t\right| \\
& \quad=\left|\int_{0}^{T_{1}} \delta_{\varepsilon}(t-\rho)[g(t)-g(\rho)] d t\right| \\
& \quad \leq \frac{c}{\varepsilon} \int_{\rho-\varepsilon}^{\rho+\varepsilon}|g(t)-g(\rho)| d t .
\end{aligned}
$$

Then

$$
\int_{0}^{T_{0}} \delta_{\varepsilon}(t-\rho) g(t) d t \longrightarrow g(\rho) \quad \text { as } \varepsilon \longrightarrow 0
$$

Let

$$
\begin{aligned}
W(\rho) & =\int_{0}^{T_{1}} \mu_{\varepsilon}(t-\rho) g(t) d t \\
& =\int_{0}^{T_{1}} d t \int_{-\infty}^{t-\rho} \delta_{\varepsilon}(\sigma) g(t) d \sigma .
\end{aligned}
$$

We observe that

$$
W^{\prime}(\rho)=-\int_{0}^{T_{1}} \delta_{\varepsilon}(t-\rho) g(t) d t .
$$

Letting $\varepsilon \rightarrow 0$, it derives that

$$
\begin{aligned}
& W^{\prime}(\rho) \longrightarrow-g(\rho), \\
& W(\rho) \longrightarrow W(0)-\int_{0}^{\rho} g(t) d t \\
& W(\tau) \longrightarrow W(0)-\int_{0}^{\tau} g(t) d t .
\end{aligned}
$$

Therefore, we have

$$
W(\rho)-W(\tau) \longrightarrow \int_{\rho}^{\tau} g(t) d t \quad \text { as } \varepsilon \longrightarrow 0 .
$$

From (63)-(69), we obtain inequality

$$
g(\rho)+c \int_{\rho}^{\tau} g(t) d t \geq g(\tau)
$$

Choosing $\rho \rightarrow 0, \tau \rightarrow t$, we get

$$
\begin{aligned}
& \int_{-\infty}^{+\infty}|\phi(0, x)-\psi(0, x)| d x \\
& \quad+c \int_{0}^{t} d t \int_{-\infty}^{+\infty}|\phi(t, x)-\psi(t, x)| d x \\
& \geq \int_{-\infty}^{+\infty}|\phi(t, x)-\psi(t, x)| d x
\end{aligned}
$$

Applying the Gronwall inequality, we complete the proof of Theorem 6. 


\section{Conflict of Interests}

The author declares that there is no conflict of interests regarding the publication of this paper.

\section{Acknowledgment}

This work is supported by National Natural Science Foundation of China (11471263).

\section{References}

[1] A. Degasperis and M. Procesi, "Asymptotic integrability," in Symmetry and Perturbation Theory, Rome, 1998, vol. 1, pp. 23-37, World Scientific, Singapore, 1999.

[2] A. Degasperis, D. Holm, and A. Hone, "A new integral equation with peakon solutions," Theoretical and Mathematical Physics, vol. 133, pp. 1461-1472, 2002.

[3] G. M. Coclite and K. H. Karlsen, "On the well-posedness of the degasperis-procesi equation," Journal of Functional Analysis, vol. 233, no. 1, pp. 60-91, 2006.

[4] G. M. Coclite and K. H. Karlsen, "On the uniqueness of discontinuous solutions to the Degasperis-Procesi equation," Journal of Differential Equations, vol. 234, no. 1, pp. 142-160, 2007.

[5] J. Escher, Y. Liu, and Z. Y. Yin, "Global weak solutions and blowup structure for the Degasperis-Procesi equation," Journal of Functional Analysis, vol. 241, no. 2, pp. 457-485, 2006.

[6] S. Y. Lai, H. Y. Yan, H. J. Chen, and Y. Wang, "The stability of local strong solutions for a shallow water equation," Journal of Inequalities and Applications, vol. 2014, article 410, 2014.

[7] S. Y. Lai and Y. H. Wu, "Global solutions and blow-up phenomena to a shallow water equation," Journal of Differential Equations, vol. 249, no. 3, pp. 693-706, 2010.

[8] J. Lenells, "Traveling wave solutions of the Degasperis-Procesi equation," Journal of Mathematical Analysis and Applications, vol. 306, no. 1, pp. 72-82, 2005.

[9] X. L. Ai and G. L. Gui, "Global well-posedness for the Cauchy problem of the viscous Degasperis-Procesi equation," Journal of Mathematical Analysis and Applications, vol. 361, no. 2, pp. 457$465,2010$.

[10] H. C. Ma, Y. D. Yu, and D. J. Ge, "New exact traveling wave solutions for the modified form of Degasperis-Procesi equation," Applied Mathematics and Computation, vol. 203, no. 2, pp. 792-798, 2008.

[11] Y. G. Fu, Z. R. Liu, and X. Y. Yang, "Orbital stability of peakons for the Degasperis-Procesi equation with strong dispersion," Nonlinear Analysis. Theory, Methods \& Applications, vol. 73, no. 2, pp. 538-546, 2010.

[12] S. N. Kružkov, "First order quasilinear equations in several independent variables," Mathematics of the USSR-Sbornik, vol. 10, no. 2, pp. 217-243, 1970. 


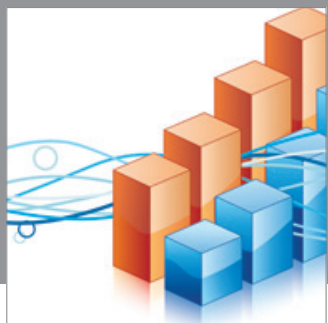

Advances in

Operations Research

mansans

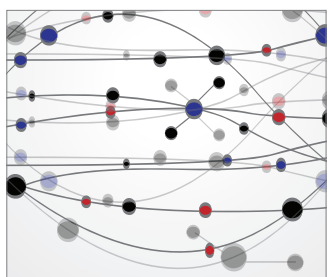

The Scientific World Journal
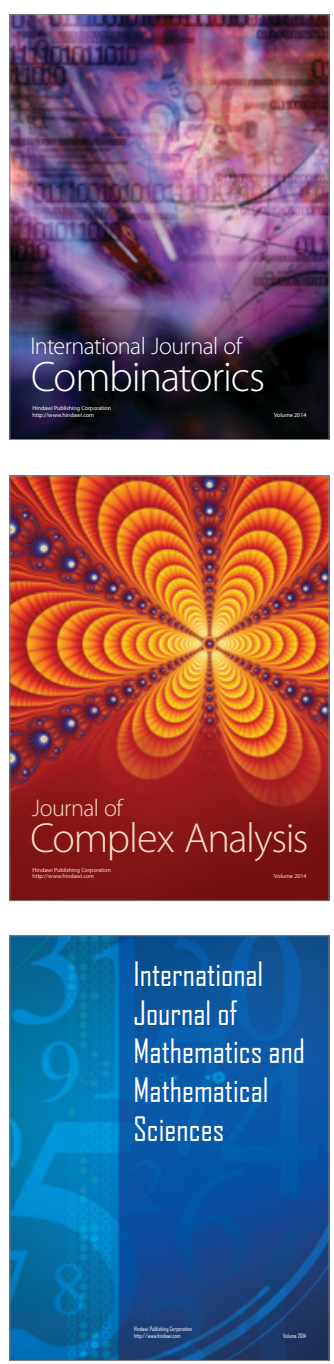
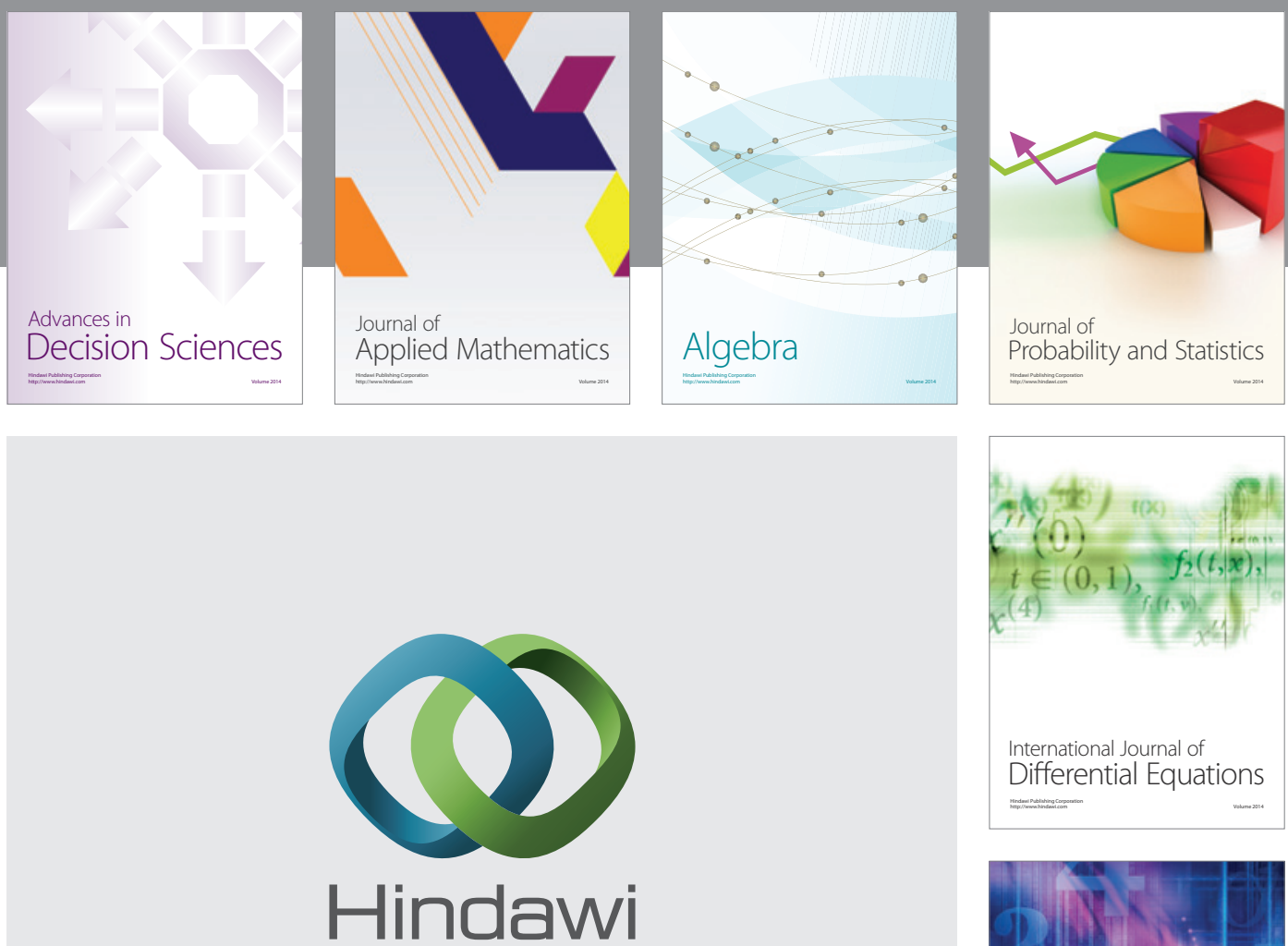

Submit your manuscripts at http://www.hindawi.com
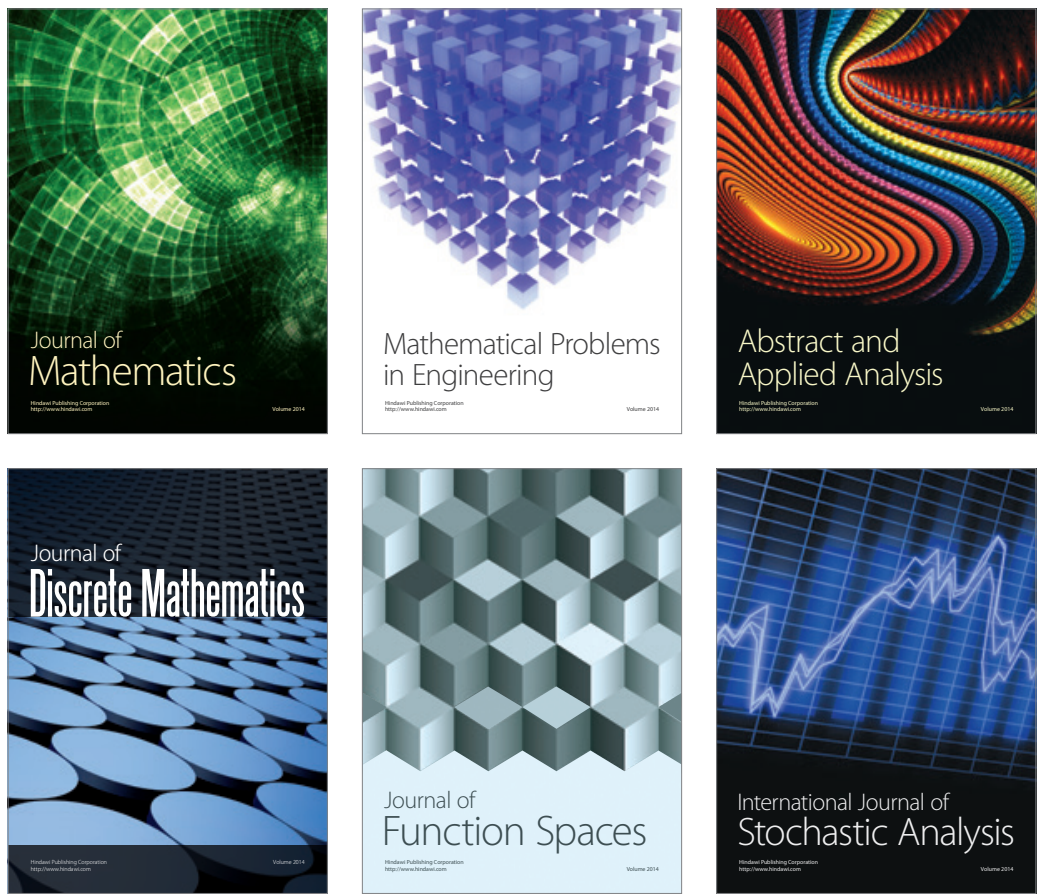

Journal of

Function Spaces

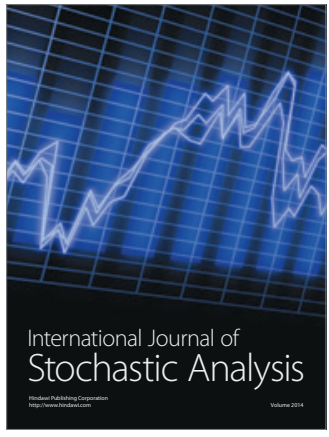

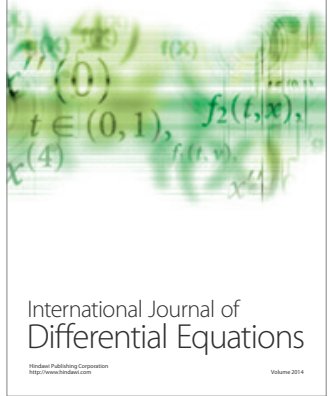
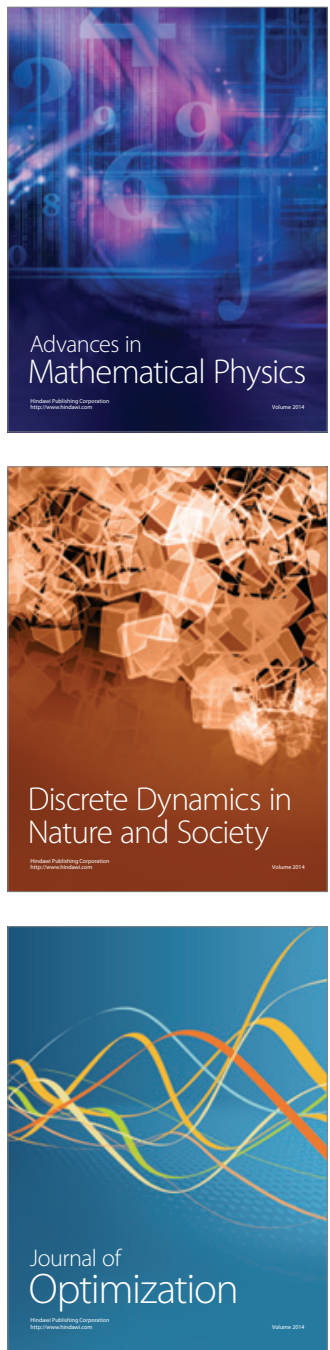